\title{
Azimuthal Asymmetries in Heavy Quark Leptoproduction as a Test of pQCD
}

\author{
N.Ya. Ivanov* \\ Yerevan Physics Institute, Alikhanian Br.2, 375036 Yerevan, Armenia
}

\begin{abstract}
We analyze the perturbative and parametric stability of the QCD predictions for the azimuthal asymmetries in heavy quark leptoproduction. At leading order, the $\cos \varphi$ asymmetry vanishes whereas the $\cos 2 \varphi$ one is of leading twist and predicted to be about $15 \%$ at energies sufficiently above the production threshold. We calculate the NLO soft-gluon corrections to $\varphi$-dependent leptoproduction to the next-to-leading logarithmic accuracy. The soft-gluon approximation provides a good description of the available exact NLO results at $Q^{2} \lesssim m^{2}$. Our analysis shows that, contrary to the production cross sections, the $\cos 2 \varphi$ asymmetry is practically insensitive to soft radiation for $Q^{2} \lesssim m^{2}$ at energies of the fixed target experiments. We conclude that the $\cos 2 \varphi$ asymmetry is well defined in pQCD: it is stable both perturbatively and parametrically, and insensitive (in the case of bottom production) to nonperturbative contributions. Measurements of the azimuthal asymmetries would provide an excellent test of pQCD applicability to heavy flavor production.
\end{abstract}

PACS: 12.38.-t, 13.60.-r, 13.88.+e

Keywords: Perturbative QCD, Heavy Flavor Leptoproduction, Azimuthal Asymmetries

\section{INTRODUCTION}

In the framework of perturbative QCD, the basic spin-averaged characteristics of heavy flavor hadro-, photo- and electroproduction are known exactly up to the next-to-leading order (NLO). During the last ten years, these NLO results have been widely used for a phenomenological description of available data (for a review see [1]). At the same time, the key question remains open: How to test the applicability of QCD at fixed order to heavy quark production? The problem is twofold. On the one hand, the NLO corrections are large; they increase the leading order (LO) predictions for both charm and bottom production cross sections by approximately a factor of two. For this reason, one could expect that higher-order corrections, as well as nonperturbative contributions, can be essential, especially for the $c$-quark case. On the other hand, it is very difficult to compare pQCD predictions for spin-averaged cross sections with experimental data directly, without additional assumptions, because of a high sensitivity of the theoretical calculations to standard uncertainties in the input QCD parameters. The total uncertainties associated with the unknown values of the heavy quark mass, $m$, the factorization and renormalization scales, $\mu_{F}$ and $\mu_{R}, \Lambda_{Q C D}$ and the parton distribution functions are so large that one can only estimate the order of magnitude of the pQCD predictions for total cross sections at fixed target energies $[2,3]$.

In recent years, the role of higher-order corrections has been extensively investigated in the framework of the soft gluon resummation formalism. For a review see Ref. [4]. Soft gluon (or threshold) resummation is based on the factorization properties of the cross section near the partonic threshold and makes it possible to resum to all orders in $\alpha_{s}$ the leading (Sudakov double) logarithms (LL) and the next-to-leading ones (NLL) [5-7]. Formally resummed cross sections are ill-defined due to the Landau pole contribution, and a few prescriptions have been proposed to avoid the renormalon ambiguities [8-10]. Unfortunately, numerical predictions for the heavy quark production cross sections can depend significantly on the choice of resummation prescription [11]. Another open question, also closely related to convergence of the perturbative series, is the role of subleading logarithms which are not, in principle, under control of the resummation procedure [11,12].

For this reason, it is of special interest to study those observables that are well-defined in pQCD. A nontrivial example of such an observable is proposed in $[13,14]$, where the single spin asymmetry (SSA) in charm and bottom

*e-mail: nikiv@uniphi.yerphi.am 
production by linearly polarized photons, $\gamma^{\uparrow}+N \rightarrow Q+X[\bar{Q}]$, was calculated. ${ }^{1}$ It was shown that, contrary to the production cross section, the single spin asymmetry in heavy flavor photoproduction is quantitatively well defined in pQCD: it is stable, both parametrically and perturbatively, and insensitive to nonperturbative corrections. Therefore, measurements of this asymmetry would provide an ideal test of pQCD. As was shown in Ref. [15], the SSA in open charm photoproduction can be measured with an accuracy of about ten percent in the approved E160/E161 experiments at SLAC [16] using the inclusive spectra of secondary (decay) leptons.

In the present paper we continue the studies of perturbatively stable observables and calculate the radiative and nonperturbative corrections to the azimuthal asymmetry (AA) in heavy quark leptoproduction:

$$
l(\ell)+N(p) \rightarrow l(\ell-q)+Q\left(p_{Q}\right)+X[\bar{Q}]\left(p_{X}\right) .
$$

In the case of unpolarized initial states and neglecting the contribution of $Z$-boson, the cross section of the reaction (1.1) may be written as

$$
\begin{aligned}
& \frac{\mathrm{d} \sigma_{l N}}{\mathrm{~d} x \mathrm{~d} Q^{2} \mathrm{~d} \varphi}=\frac{\alpha_{e m}}{(2 \pi)^{2}} \frac{1}{x Q^{2}}\left\{\left[1+(1-y)^{2}\right] \sigma_{T}\left(x, Q^{2}\right)+2(1-y) \sigma_{L}\left(x, Q^{2}\right)\right. \\
&\left.+2(1-y) \sigma_{A}\left(x, Q^{2}\right) \cos 2 \varphi+(2-y) \sqrt{1-y} \sigma_{I}\left(x, Q^{2}\right) \cos \varphi\right\} .
\end{aligned}
$$

The kinematic variables are defined by

$$
\begin{aligned}
\bar{S}=(\ell+p)^{2}, & Q^{2}=-q^{2}, & x=\frac{Q^{2}}{2 p \cdot q}, \\
y=\frac{p \cdot q}{p \cdot \ell}, & Q^{2}=x y \bar{S}, & \rho=\frac{4 m^{2}}{\bar{S}} .
\end{aligned}
$$

In Eq. (1.2), $\sigma_{T}\left(\sigma_{L}\right)$ is the usual $\gamma^{*} N$ cross section describing heavy quark production by a transverse (longitudinal) virtual photon. The third cross section, $\sigma_{A}$, comes about from interference between transverse states and is responsible for the SSA which occurs in real photoproduction using linearly polarized photons [13-15]. The fourth cross section, $\sigma_{I}$, originates from interference between longitudinal and transverse components [17]. In the nucleon rest frame, the azimuth $\varphi$ is the angle between the lepton scattering plane and the heavy quark production plane, defined by the exchanged photon and the detected quark $Q$ (see Fig. 1). The covariant definition of $\varphi$ is

$$
\begin{aligned}
\cos \varphi & =\frac{r \cdot n}{\sqrt{-r^{2}} \sqrt{-n^{2}}}, & \sin \varphi & =\frac{Q^{2} \sqrt{1 / x^{2}+4 m_{N}^{2} / Q^{2}}}{2 \sqrt{-r^{2}} \sqrt{-n^{2}}} n \cdot \ell, \\
r^{\mu} & =\varepsilon^{\mu \nu \alpha \beta} p_{\nu} q_{\alpha} \ell_{\beta}, & n^{\mu} & =\varepsilon^{\mu \nu \alpha \beta} p_{\nu} q_{\alpha} p_{Q \beta} .
\end{aligned}
$$

In Eqs. (1.3) and (1.4), $m$ and $m_{N}$ are the masses of the heavy quark and the target, respectively.

In leading order $\mathrm{pQCD}$, the $\cos \varphi$ dependence vanishes, $\sigma_{I}^{\text {Born }}\left(x, Q^{2}\right)=0$. For this reason, in this paper we restrict ourselves to the azimuthal asymmetry, $A\left(\rho, x, Q^{2}\right)$, associated with the $\cos 2 \varphi$ distribution:

$$
A\left(\rho, x, Q^{2}\right)=\frac{\mathrm{d}^{3} \sigma_{l N}(\varphi=0)+\mathrm{d}^{3} \sigma_{l N}(\varphi=\pi)-2 \mathrm{~d}^{3} \sigma_{l N}(\varphi=\pi / 2)}{\mathrm{d}^{3} \sigma_{l N}(\varphi=0)+\mathrm{d}^{3} \sigma_{l N}(\varphi=\pi)+2 \mathrm{~d}^{3} \sigma_{l N}(\varphi=\pi / 2)}=\frac{\varepsilon \sigma_{A}\left(x, Q^{2}\right)}{\sigma_{T}\left(x, Q^{2}\right)+\varepsilon \sigma_{L}\left(x, Q^{2}\right)},
$$

where $\varepsilon=\frac{2(1-y)}{1+(1-y)^{2}}$ and $\mathrm{d}^{3} \sigma_{l N}(\varphi) \equiv \frac{\mathrm{d}^{3} \sigma_{l N}}{\mathrm{~d} x \mathrm{~d} Q^{2} \mathrm{~d} \varphi}\left(\rho, x, Q^{2}, \varphi\right)$. Note that the asymmetry defined by Eq. (1.6) is simply related to the mean value of $\cos 2 \varphi$ :

\footnotetext{
${ }^{1}$ The well-known examples are the shapes of differential cross sections of heavy flavor production which are sufficiently stable under radiative corrections.
} 


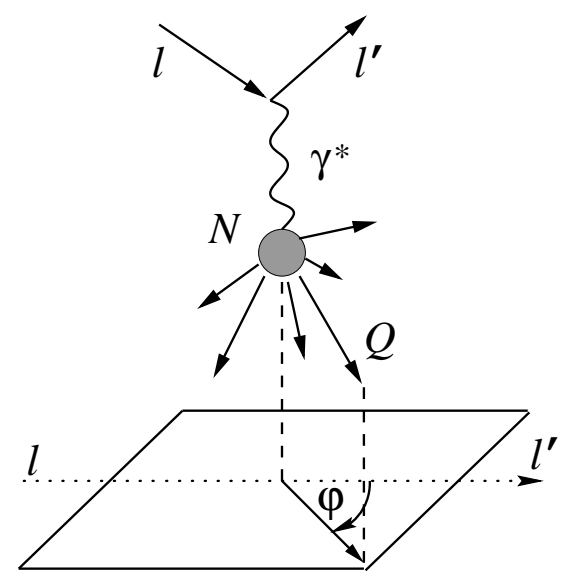

FIG. 1. Definition of the azimuthal angle $\varphi$ in the nucleon rest frame.

$$
A\left(\rho, x, Q^{2}\right)=2\langle\cos 2 \varphi\rangle\left(\rho, x, Q^{2}\right), \quad\langle\cos 2 \varphi\rangle\left(\rho, x, Q^{2}\right)=\frac{\int_{0}^{2 \pi} \mathrm{d} \varphi \cos 2 \varphi \frac{\mathrm{d}^{3} \sigma_{l N}}{\mathrm{~d} x \mathrm{~d} Q^{2} \mathrm{~d} \varphi}\left(\rho, x, Q^{2}, \varphi\right)}{\int_{0}^{2 \pi} \mathrm{d} \varphi \frac{\mathrm{d}^{3} \sigma_{l N}}{\mathrm{~d} x \mathrm{~d} Q^{2} \mathrm{~d} \varphi}\left(\rho, x, Q^{2}, \varphi\right)} .
$$

In this paper, we calculate the NLO corrections to the $\cos 2 \varphi$ asymmetry to the next-to-leading logarithmic accuracy (so-called soft-gluon approximation). Also we analyze the nonperturbative contributions to the AA due to the gluon transverse motion in the target. Our main results can be formulated as follows:

- The azimuthal asymmetry defined by Eq. (1.6) is of leading twist; at energies sufficiently above the production threshold, it is predicted to be about $15 \%$ for both charm and bottom quark production.

- The soft-gluon approximation provides a good description of the available exact NLO results on leptoproduction in the region of relatively low virtualities, $Q^{2} \lesssim m^{2}$; when $Q^{2} \gg m^{2}$, the quality of the NLL approximation becomes worse.

- Contrary to the production cross sections, the $\cos 2 \varphi$ asymmetry in azimuthal distributions of both charm and bottom quark is practically insensitive to radiative corrections at $Q^{2} \lesssim m^{2}$. This implies that large soft-gluon contributions to the $\varphi$-dependent and $\varphi$-integrated cross sections cancel each other in Eq. (1.6) with a good accuracy.

- pQCD predictions for the $\cos 2 \varphi$ asymmetry are parametrically stable; to within few percent, they are insensitive to standard uncertainties in the QCD input parameters: $\mu_{R}, \mu_{F}, \Lambda_{Q C D}$ and in the gluon distribution function.

- Nonperturbative corrections to the $b$-quark azimuthal asymmetry due to the gluon transverse motion in the target are negligible. Because of the smallness of the $c$-quark mass, the analogous corrections to $A\left(\rho, x, Q^{2}\right)$ in the charm case are larger; they are of the order of $20 \%$ at $Q^{2} \lesssim m^{2}$.

We conclude that, in contrast with the production cross sections, the $\cos 2 \varphi$ asymmetry in heavy quark leptoproduction, $A\left(\rho, x, Q^{2}\right)$, is an observable quantitatively well defined in pQCD: it is stable, both parametrically and perturbatively, and insensitive (in the case of bottom production) to nonperturbative corrections. Measurements of the AA in bottom production would provide an ideal test of the conventional parton model based on pQCD.

Concerning the experimental aspects, azimuthal asymmetries in bottom production can, in principle, be measured at HERA using the angular distributions of secondary (decay) leptons [15]. AAs in charm leptoproduction can also be measured in the COMPASS and HERMES experiments. Due to the relatively low $c$-quark mass, data on the $D$-meson azimuthal distributions would make it possible to clarify the role of subleading twist contributions. 
The paper is organized as follows. In Section II we analyze the LO and NLO parton level predictions for $\varphi$ dependent leptoproduction of heavy flavor in the single-particle inclusive kinematics. We check the quality of the soft-gluon approach against available exact results and discuss the region of applicability of the NLL approximation. Hadron level predictions for $A\left(\rho, x, Q^{2}\right)$ are given in Section III. We consider in detail the pQCD contributions and nonperturbative corrections to the $\cos 2 \varphi$ asymmetry at the HERMES, SLAC, COMPASS and HERA energies.

\section{PARTONIC CROSS SECTIONS}

\section{A. Born level predictions}

At leading order, $\mathcal{O}\left(\alpha_{e m} \alpha_{s}\right)$, the only partonic subprocess which is responsible for heavy quark leptoproduction is the two-body photon-gluon fusion:

$$
\gamma^{*}(q)+g\left(k_{g}\right) \rightarrow Q\left(p_{Q}\right)+\bar{Q}\left(p_{\bar{Q}}\right)
$$

The $\gamma^{*} g$ cross sections, $\hat{\sigma}_{k}^{\text {Born }}(k=T, L, A, I)$, corresponding to the Born diagrams are $[18,19]$ :

$$
\begin{aligned}
& \hat{\sigma}_{T}^{\text {Born }}(\hat{\rho}, \hat{x})=\frac{\pi e_{Q}^{2} \alpha_{e m} \alpha_{s}}{2 m^{2}} \hat{\rho}\left\{\left[(1-\hat{x})^{2}+\hat{x}^{2}+\hat{\rho}(1-\hat{x}-\hat{\rho} / 2)\right] \ln \frac{1+\beta}{1-\beta}\right. \\
& \left.-\left[(1-\hat{x})^{2}-\hat{x}(2-3 \hat{x})+(1-\hat{x}) \hat{\rho}\right] \beta\right\}, \\
& \hat{\sigma}_{L}^{\text {Born }}(\hat{\rho}, \hat{x})=\frac{\pi e_{Q}^{2} \alpha_{e m} \alpha_{s}}{m^{2}} \hat{\rho} \hat{x}\left\{-\hat{\rho} \ln \frac{1+\beta}{1-\beta}+2(1-\hat{x}) \beta\right\}, \\
& \hat{\sigma}_{A}^{\text {Born }}(\hat{\rho}, \hat{x})=\frac{\pi e_{Q}^{2} \alpha_{e m} \alpha_{s}}{2 m^{2}} \hat{\rho}^{2}\left\{(1-2 \hat{x}-\hat{\rho} / 2) \ln \frac{1+\beta}{1-\beta}-(1-\hat{x})(1-2 \hat{x} / \hat{\rho}) \beta\right\}, \\
& \hat{\sigma}_{I}^{\text {Born }}(\hat{\rho}, \hat{x})=0 .
\end{aligned}
$$

In Eqs. (2.2), $e_{Q}$ is the quark charge in units of electromagnetic coupling constant and we use the following definition of partonic kinematic variables:

$$
\begin{aligned}
s=\left(q+k_{g}\right)^{2}, & \hat{x}=\frac{Q^{2}}{2 q \cdot k_{g}}, \\
\beta=\sqrt{1-\frac{4 m^{2}}{s}}, & \hat{\rho}=\frac{4 m^{2}}{s+Q^{2}} .
\end{aligned}
$$

Note that the $\cos \varphi$ dependence vanishes due to the $Q \leftrightarrow \bar{Q}$ symmetry which, at leading order, requires invariance under $\varphi \rightarrow \varphi+\pi[20]$.

The hadron level cross sections, $\sigma_{k}\left(x, Q^{2}\right)(k=T, L, A, I)$, have the form

$$
\sigma_{k}(x, \xi)=\int_{x+4 x / \xi}^{1} \mathrm{~d} z g\left(z, \mu_{F}\right) \hat{\sigma}_{k}\left(\frac{4 x}{z \xi}, \frac{x}{z}\right), \quad k_{g}=z p, \quad \xi=\frac{Q^{2}}{m^{2}}
$$

where $g\left(z, \mu_{F}\right)$ describes gluon density in a nucleon $N$ evaluated at a factorization scale $\mu_{F}$. The partonic cross sections, $\hat{\sigma}_{k}$, are functions of $\hat{\rho}$ and $\hat{x}$ defined by Eq. (2.3).

\section{B. Soft-gluon corrections at NLO}

To take into account the NLO contributions, one needs to calculate the virtual $\mathcal{O}\left(\alpha_{e m} \alpha_{s}^{2}\right)$ corrections to the Born process (2.1) and the real gluon emission: 


$$
\gamma^{*}(q)+g\left(k_{g}\right) \rightarrow Q\left(p_{Q}\right)+\bar{Q}\left(p_{\bar{Q}}\right)+g\left(p_{g}\right) .
$$

The partonic invariants describing the single-particle inclusive (1PI) kinematics are

$$
\begin{aligned}
s^{\prime}=2 q \cdot k_{g}=s+Q^{2}=z S^{\prime}, & t_{1}=\left(k_{g}-p_{Q}\right)^{2}-m^{2}=z T_{1}, \\
s_{4}=s^{\prime}+t_{1}+u_{1}, & u_{1}=\left(q-p_{Q}\right)^{2}-m^{2}=U_{1},
\end{aligned}
$$

where $s_{4}$ measures the inelasticity of the reaction (2.5). The corresponding 1PI hadron level variables describing the reaction (1.1) are

$$
\begin{aligned}
S^{\prime}=2 q \cdot p=S+Q^{2}, & T_{1}=\left(p-p_{Q}\right)^{2}-m^{2}, \\
S_{4}=S^{\prime}+T_{1}+U_{1}, & U_{1}=\left(q-p_{Q}\right)^{2}-m^{2} .
\end{aligned}
$$

We neglect the photon-(anti)quark fusion subprocesses. This is justified as their contributions vanish at LO and are small at NLO [21].

The exact NLO calculations of the unpolarized heavy quark production in $\gamma g$ [22,23], $\gamma^{*} g$ [21], and $g g$ [24,25] collisions show that, near the partonic threshold, a strong logarithmic enhancement of the cross sections takes place in the collinear, $\vec{p}_{g, T} \rightarrow 0$, and soft, $\vec{p}_{g} \rightarrow 0$, limits. This threshold (or soft-gluon) enhancement has universal nature in the perturbation theory and originates from incomplete cancellation of the soft and collinear singularities between the loop and the bremsstrahlung contributions. Large leading and next-to-leading threshold logarithms can be resummed to all orders of perturbative expansion using the appropriate evolution equations [5-7]. The analytic results for the resummed cross sections are ill-defined due to the Landau pole in the coupling strength $\alpha_{s}$. However, if one considers the obtained expressions as generating functionals of the perturbative theory and re-expands them at fixed order in $\alpha_{s}$, no divergences associated with the Landau pole are encountered.

Soft-gluon resummation for the photon-gluon fusion has been performed in Ref. [26] and checked in Refs. [27,14]. To NLL accuracy, the perturbative expansion for the partonic cross sections, $\mathrm{d}^{2} \hat{\sigma}_{k} / \mathrm{d} t_{1} \mathrm{~d} u_{1}(k=T, L, A, I)$, can be written in a factorized form as

$$
s^{\prime 2} \frac{\mathrm{d}^{2} \hat{\sigma}_{k}}{\mathrm{~d} t_{1} \mathrm{~d} u_{1}}\left(s^{\prime}, t_{1}, u_{1}\right)=B_{k}^{\text {Born }}\left(s^{\prime}, t_{1}, u_{1}\right)\left\{\delta\left(s^{\prime}+t_{1}+u_{1}\right)+\sum_{n=1}^{\infty}\left(\frac{\alpha_{s} C_{A}}{\pi}\right)^{n} K^{(n)}\left(s^{\prime}, t_{1}, u_{1}\right)\right\},
$$

with the Born level distributions $B_{k}^{\text {Born }}$ given by

$$
\begin{aligned}
& B_{T}^{\text {Born }}\left(s^{\prime}, t_{1}, u_{1}\right)=\pi e_{Q}^{2} \alpha_{e m} \alpha_{s}\left[\frac{t_{1}}{u_{1}}+\frac{u_{1}}{t_{1}}+4\left(\frac{s}{s^{\prime}}-\frac{m^{2} s^{\prime}}{t_{1} u_{1}}\right)\left(\frac{s^{\prime}\left(m^{2}-Q^{2} / 2\right)}{t_{1} u_{1}}+\frac{Q^{2}}{s^{\prime}}\right)\right], \\
& B_{L}^{\text {Born }}\left(s^{\prime}, t_{1}, u_{1}\right)=\pi e_{Q}^{2} \alpha_{e m} \alpha_{s}\left[\frac{8 Q^{2}}{s^{\prime}}\left(\frac{s}{s^{\prime}}-\frac{m^{2} s^{\prime}}{t_{1} u_{1}}\right)\right], \\
& B_{A}^{\text {Born }}\left(s^{\prime}, t_{1}, u_{1}\right)=\pi e_{Q}^{2} \alpha_{e m} \alpha_{s}\left[4\left(\frac{s}{s^{\prime}}-\frac{m^{2} s^{\prime}}{t_{1} u_{1}}\right)\left(\frac{m^{2} s^{\prime}}{t_{1} u_{1}}+\frac{Q^{2}}{s^{\prime}}\right)\right], \\
& B_{I}^{\text {Born }}\left(s^{\prime}, t_{1}, u_{1}\right)=\pi e_{Q}^{2} \alpha_{e m} \alpha_{s}\left[4 \sqrt{Q^{2}}\left(\frac{t_{1} u_{1} s}{s^{\prime 2}}-m^{2}\right)^{1 / 2} \frac{t_{1}-u_{1}}{t_{1} u_{1}}\left(1-\frac{2 Q^{2}}{s^{\prime}}-\frac{2 m^{2} s^{\prime}}{t_{1} u_{1}}\right)\right] .
\end{aligned}
$$

Note that the functions $K^{(n)}\left(s^{\prime}, t_{1}, u_{1}\right)$ in Eq. (2.8) originate from the collinear and soft limits. Since the azimuthal angle $\varphi$ is the same for both $\gamma^{*} g$ and $Q \bar{Q}$ center-of-mass systems in these limits, the functions $K^{(n)}\left(s^{\prime}, t_{1}, u_{1}\right)$ are also the same for all $k=T, L, A, I$. At NLO, the soft-gluon corrections to NLL accuracy in the $\overline{\mathrm{MS}}$ scheme are

$$
\begin{aligned}
K^{(1)}\left(s^{\prime}, t_{1}, u_{1}\right)= & 2\left[\frac{\ln \left(s_{4} / m^{2}\right)}{s_{4}}\right]_{+}-\left[\frac{1}{s_{4}}\right]_{+}\left\{1+\ln \left(\frac{u_{1}}{t_{1}}\right)-\left(1-\frac{2 C_{F}}{C_{A}}\right)\left(1+\operatorname{Re} L_{\beta}\right)+\ln \left(\frac{\mu^{2}}{m^{2}}\right)\right\} \\
& +\delta\left(s_{4}\right) \ln \left(\frac{-u_{1}}{m^{2}}\right) \ln \left(\frac{\mu^{2}}{m^{2}}\right),
\end{aligned}
$$


where we use $\mu=\mu_{F}=\mu_{R}$. In Eq. (2.10), $C_{A}=N_{c}$ and $C_{F}=\left(N_{c}^{2}-1\right) /\left(2 N_{c}\right)$, where $N_{c}$ is number of colors, while $L_{\beta}=\left(1-2 m^{2} / s\right)\{\ln [(1-\beta) /(1+\beta)]+\mathrm{i} \pi\}$. The single-particle inclusive "plus" distributions are defined by

$$
\left[\frac{\ln ^{l}\left(s_{4} / m^{2}\right)}{s_{4}}\right]_{+}=\lim _{\epsilon \rightarrow 0}\left\{\frac{\ln ^{l}\left(s_{4} / m^{2}\right)}{s_{4}} \theta\left(s_{4}-\epsilon\right)+\frac{1}{l+1} \ln ^{l+1}\left(\frac{\epsilon}{m^{2}}\right) \delta\left(s_{4}\right)\right\} .
$$

For any sufficiently regular test function $h\left(s_{4}\right)$, Eq. (2.11) gives

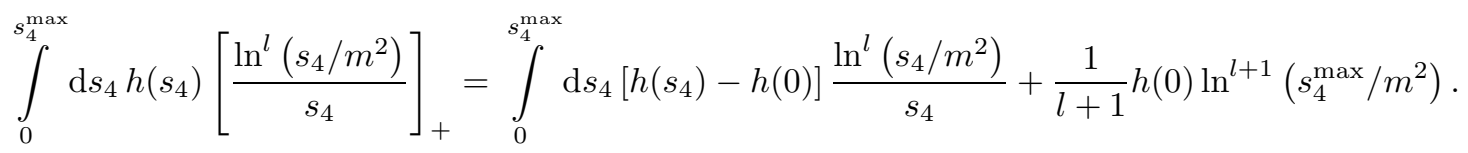

In Eq. (2.10), we have preserved the NLL terms for the scale-dependent logarithms too. We have checked that the results (2.9) and (2.10) agree to NLL accuracy with the exact $\mathcal{O}\left(\alpha_{e m} \alpha_{s}^{2}\right)$ calculations of the photon-gluon cross sections $\hat{\sigma}_{T}$ and $\hat{\sigma}_{L}$ given in Ref. [21].

To perform a numerical investigation of the results (2.9) and (2.10), it is convenient to introduce for the fully inclusive (integrated over $t_{1}$ and $\left.u_{1}\right)$ cross sections, $\hat{\sigma}_{k}(k=T, L, A, I)$, the dimensionless coefficient functions $c_{k}^{(n, l)}$,

$$
\hat{\sigma}_{k}\left(\eta, \xi, \mu^{2}\right)=\frac{e_{Q}^{2} \alpha_{e m} \alpha_{s}\left(\mu^{2}\right)}{m^{2}} \sum_{n=0}^{\infty}\left(4 \pi \alpha_{s}\left(\mu^{2}\right)\right)^{n} \sum_{l=0}^{n} c_{k}^{(n, l)}(\eta, \xi) \ln ^{l}\left(\frac{\mu^{2}}{m^{2}}\right)
$$

where the variable $\eta$ measures the distance to the partonic threshold:

$$
\eta=\frac{s}{4 m^{2}}-1, \quad \xi=\frac{Q^{2}}{m^{2}} .
$$

Concerning the NLO scale-independent coefficient functions, only $c_{T}^{(1,0)}$ and $c_{L}^{(1,0)}$ are known exactly [21,28]. As to the $\mu$-dependent coefficients, they can by calculated explicitly using the renormalization group equation:

$$
\frac{\mathrm{d} \hat{\sigma}_{k}\left(s^{\prime}, Q^{2}, \mu^{2}\right)}{\mathrm{d} \ln \mu^{2}}=-\int_{z_{\min }}^{1} \mathrm{~d} z \hat{\sigma}_{k}\left(z s^{\prime}, Q^{2}, \mu^{2}\right) P_{g g}(z)
$$

where $z_{\text {min }}=\left(4 m^{2}+Q^{2}\right) / s^{\prime}, \hat{\sigma}_{k}\left(s^{\prime}, Q^{2}, \mu\right)$ are the cross sections resummed to all orders in $\alpha_{s}$ and $P_{g g}(z)$ is the corresponding (resummed) Altarelli-Parisi gluon-gluon splitting function. Expanding Eq. (2.15) in $\alpha_{s}$, one can find $[26,14]$

$$
c_{k}^{(1,1)}\left(s^{\prime}, \xi\right)=\frac{1}{4 \pi^{2}} \int_{z_{\min }}^{1} \mathrm{~d} z\left[b_{2} \delta(1-z)-P_{g g}^{(0)}(z)\right] c_{k}^{(0,0)}\left(z s^{\prime}, \xi\right),
$$

where $b_{2}=\left(11 C_{A}-2 n_{f}\right) / 12$ is the first coefficient of the $\beta\left(\alpha_{s}\right)$-function expansion and $n_{f}$ is the number of active quark flavors. The one-loop gluon splitting function is [29]:

$$
P_{g g}^{(0)}(z)=\lim _{\epsilon \rightarrow 0}\left\{\left(\frac{z}{1-z}+\frac{1-z}{z}+z(1-z)\right) \theta(1-z-\epsilon)+\delta(1-z) \ln \epsilon\right\} C_{A}+b_{2} \delta(1-z) .
$$

With Eq. (2.16) in hand, we are able to check the quality of the NLL approximation against exact answers. In Figs. 2 and 3 we plot the functions $c_{T}^{(n, l)}(\eta, \xi)$ and $c_{A}^{(n, l)}(\eta, \xi)(n, l=0,1)$ at $\xi=10^{-2}$ and $\xi=3.16$, respectively. Predictions of the NLL approximation (2.10) are given by dotted curves. The available exact results are given by solid lines. One can see a reasonable agreement up to energies $\eta \approx 2$. As to the $Q^{2}$-dependence, we have found that the soft-gluon approach reproduces satisfactorily the exact results at $\xi \lesssim 1$. At high values of $\xi, Q^{2} \gg m^{2}$, the quality of the NLL approximation becomes worse. ${ }^{2}$

\footnotetext{
${ }^{2}$ Our analysis shows that the same situation takes also place for the energy and $Q^{2}$ behavior of the functions $c_{L}^{(1, l)}(\eta, \xi)$, $l=0,1$. We do not give corresponding plots for $c_{L}^{(1, l)}(\eta, \xi)$ because the contribution of the longitudinal cross section to the $\cos 2 \varphi$ asymmetry is small numerically.
} 

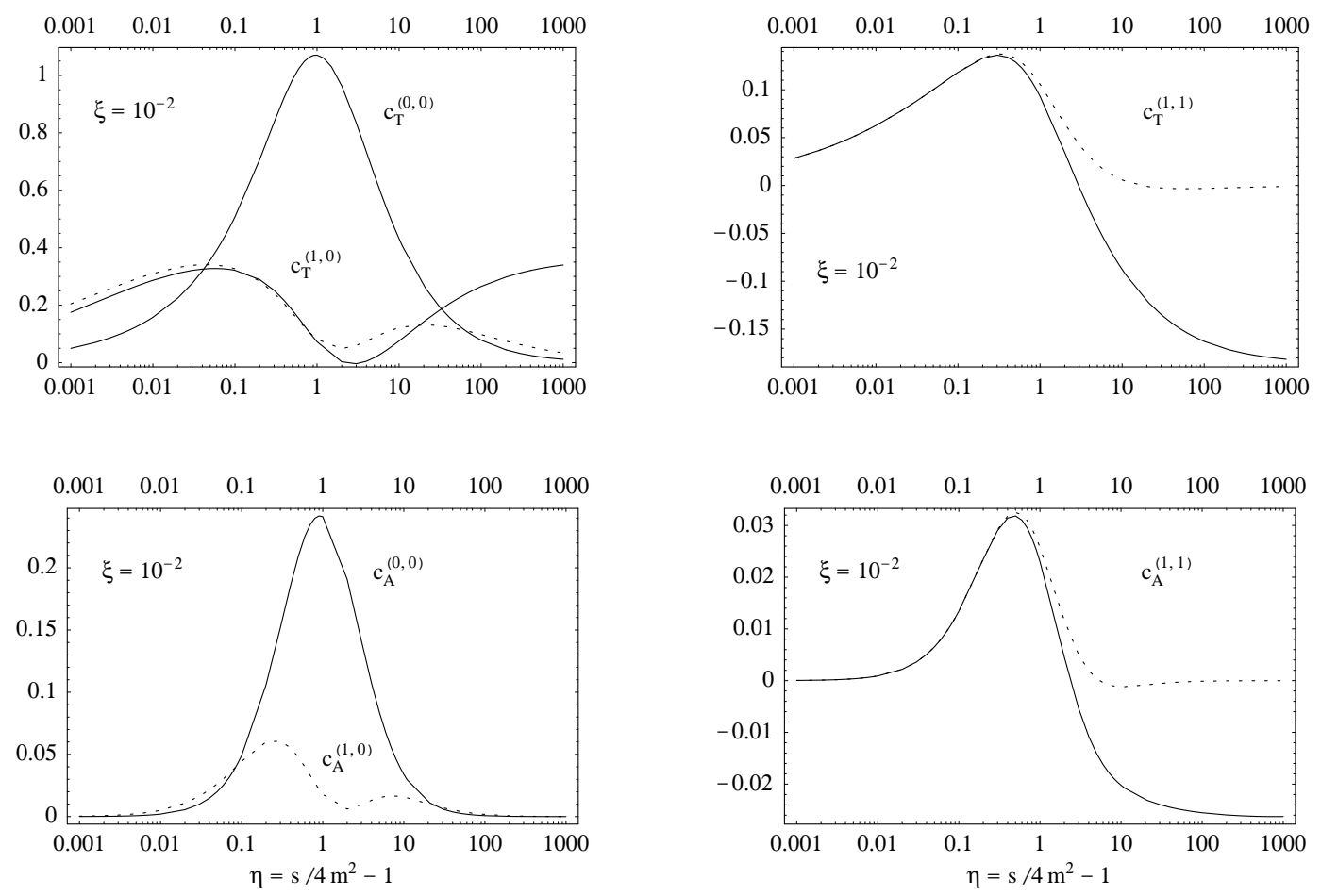

FIG. 2. $c_{T}^{(k, l)}(\eta, \xi)$ and $c_{A}^{(k, l)}(\eta, \xi)$ coefficient functions at $\xi=10^{-2}$. Plotted are the available exact results (solid lines) and the NLL approximation (dotted lines).
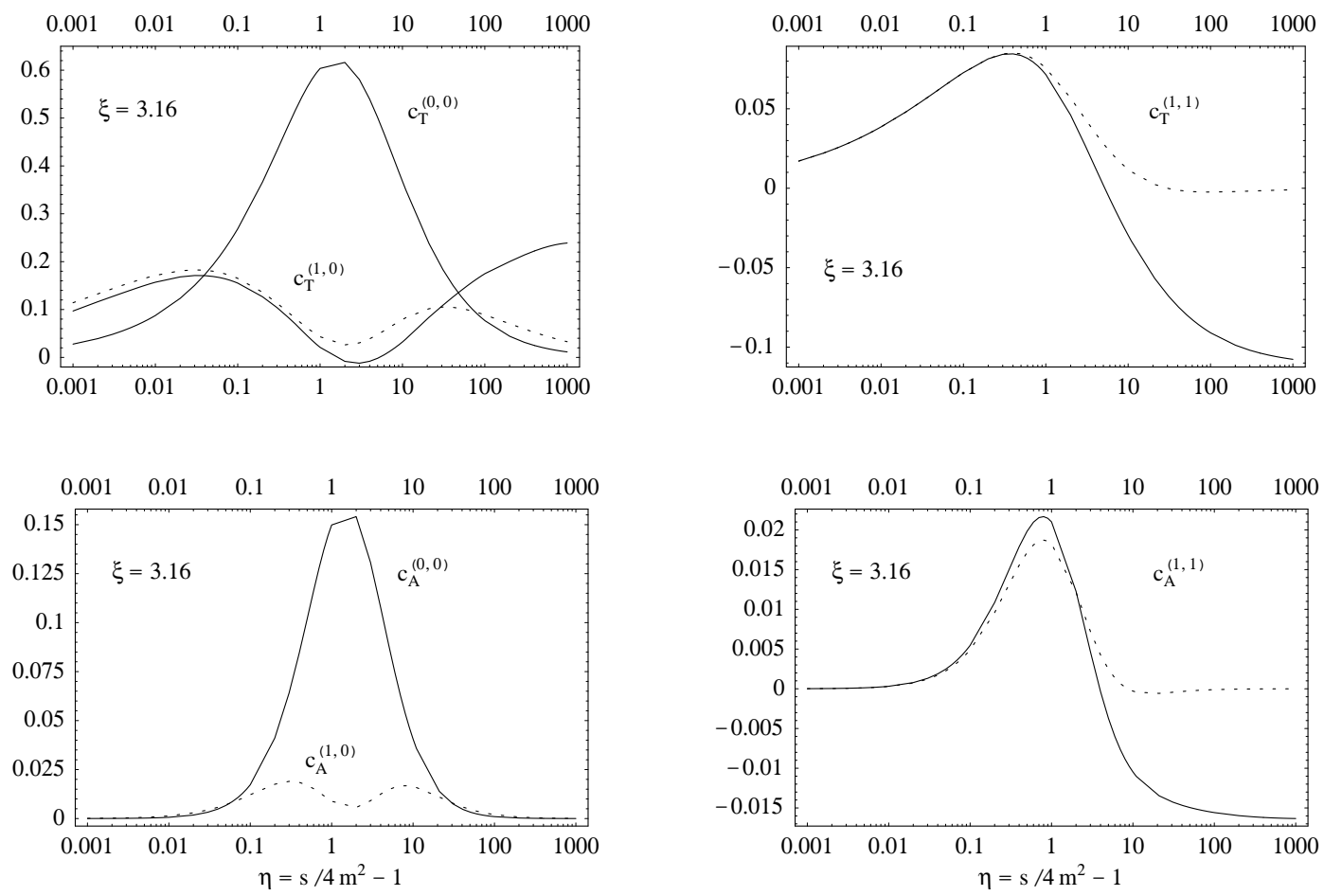

FIG. 3. $c_{T}^{(k, l)}(\eta, \xi)$ and $c_{A}^{(k, l)}(\eta, \xi)$ coefficient functions at $\xi=3.16$. Plotted are the available exact results (solid lines) and the NLL approximation (dotted lines). 


\section{HADRON LEVEL RESULTS}

\section{A. pQCD predictions}

Let us now analyze the impact of the approximate NLO perturbative corrections on the AA at hadron level. We will consider the parameters $A\left(Q^{2}\right), A(x)$ and $A(y)$,

$$
\begin{gathered}
A\left(Q^{2}\right)=\frac{2 \int_{0}^{2 \pi} \mathrm{d} \varphi \cos 2 \varphi \frac{\mathrm{d}^{2} \sigma_{l N}}{\mathrm{~d} Q^{2} \mathrm{~d} \varphi}\left(\rho, Q^{2}, \varphi\right)}{\int_{0}^{2 \pi} \mathrm{d} \varphi \frac{\mathrm{d}^{2} \sigma_{l N}}{\mathrm{~d} Q^{2} \mathrm{~d} \varphi}\left(\rho, Q^{2}, \varphi\right)}, \quad A(x)=\frac{2 \int_{0}^{2 \pi} \mathrm{d} \varphi \cos 2 \varphi \frac{\mathrm{d}^{2} \sigma_{l N}}{\mathrm{~d} x \mathrm{~d} \varphi}(\rho, x, \varphi)}{\int_{0}^{2 \pi} \mathrm{d} \varphi \frac{\mathrm{d}^{2} \sigma_{l N}}{\mathrm{~d} x \mathrm{~d} \varphi}(\rho, x, \varphi)}, \\
A(y)=\frac{2 \int_{0}^{2 \pi} \mathrm{d} \varphi \cos 2 \varphi \frac{\mathrm{d}^{2} \sigma_{l N}}{\mathrm{~d} y \mathrm{~d} \varphi}(\rho, y, \varphi)}{\int_{0}^{2 \pi} \mathrm{d} \varphi \frac{\mathrm{d}^{2} \sigma_{l N}}{\mathrm{~d} y \mathrm{~d} \varphi}(\rho, y, \varphi)},
\end{gathered}
$$

which describe the dependence of the $\cos 2 \varphi$ asymmetry on $Q^{2}$, Bjorken $x$ and $y$, respectively. Unless otherwise stated, the CTEQ5M [30] parametrization of the gluon distribution function is used. The default values of the charm and bottom mass are $m_{c}=1.5 \mathrm{GeV}$ and $m_{b}=4.75 \mathrm{GeV}, \Lambda_{3}=260 \mathrm{MeV}$ and $\Lambda_{4}=200 \mathrm{MeV}$. For the factorization scale we use $\mu_{F}=\sqrt{m_{b}^{2}+Q^{2} / 4}$ in the case of bottom production and $\mu_{F}=2 \sqrt{m_{c}^{2}+Q^{2} / 4}$ in the charm case [1].

Our results for the $Q^{2}$ - and $x$-distributions of the AA in charm leptoproduction at several values of initial energy are presented in Fig. 4 and Fig. 5, respectively. The LO and NLO predictions are given by solid and dotted lines, correspondingly. The lines with label " 1 " correspond to $\rho_{1}=0.2, " 2$ " $\rightarrow \rho_{2}=0.1$, " 3 " $\rightarrow \rho_{3}=0.05$ and " 4 " $\rightarrow \rho_{4}=0.025$, where $\rho=4 m^{2} / \bar{S}$. So, in the charm case, we have: $E_{1}=24 \mathrm{GeV}, E_{2}=47 \mathrm{GeV}, E_{3}=95 \mathrm{GeV}$ and $E_{4}=190 \mathrm{GeV}$, where $E$ is the lepton energy in the lab (nucleon rest) frame: $E=\left(\bar{S}-m_{N}^{2}\right) /\left(2 m_{N}\right)$. The $y$-distribution of the asymmetry at the COMPASS energies is given in Fig. 6. In Fig. 7 we plot the $A\left(Q^{2}\right), A(x)$ and $A(y)$ distributions of the asymmetry in bottom production at the same values of $\rho_{i}=\{0.2,0.1,0.05,0.025\}$ which correspond to the following set of initial energies: $E_{i}=\{240,480,960,1920\}$ in units of $\mathrm{GeV}$.

Our calculations given in Figs. 4-7 represent the central result of this paper. One can see from Figs. 4 and 7 that soft-gluon corrections to $A\left(Q^{2}\right)$ are about few percent at not large $Q^{2} \lesssim m^{2}$. At fixed values of $x$, the kinematical restriction $Q^{2} \lesssim m^{2}$ leads to $x \lesssim m^{2} / \bar{S}$. For this reason, radiative correction to $A(x)$ are small in the region of $x \lesssim \rho$ (see Figs. 5 and 7 ). For comparison, we plot in Fig. 8 the so-called $K$-factors for $\varphi$-integrated cross sections: $K\left(Q^{2}\right)=\frac{\mathrm{d} \sigma_{l N}^{\mathrm{NLO}}}{\mathrm{d} Q^{2}} / \frac{\mathrm{d} \sigma_{l N}^{\mathrm{LO}}}{\mathrm{d} Q^{2}}$ and $K(x)=\frac{\mathrm{d} \sigma_{l N}^{\mathrm{NLO}}}{\mathrm{d} x} / \frac{\mathrm{d} \sigma_{l N}^{\mathrm{LO}}}{\mathrm{d} x}$. One can see that large soft-gluon corrections to the production cross sections practically (to within few percent) do not affect the Born predictions for the $\cos 2 \varphi$ asymmetry at $Q^{2} \lesssim m^{2}$ and $x \lesssim \rho$

At fixed values of $y$, the allowed region of $Q^{2}$ is $m_{l}^{2} y^{2} /(1-y) \leq Q^{2} \leq y \bar{S}-4 m^{2}$, where $m_{l}$ is the initial lepton mass. Since production cross sections rapidly vanish with growth of $Q^{2}$, practically whole contribution to $A(y)$ originates from the low $-Q^{2}$ region. For this reason, radiative corrections to $A(y)$ are negligible practically in the whole region of $y$ (see Figs. 6 and 7 ).

Let us now discuss the region of applicability of the adopted soft-gluon approximation. As noted in previous Section, soft radiation reproduces satisfactorily the existing exact NLO results when $\xi$ is not large, $Q^{2} \lesssim m^{2}$. At large $Q^{2} \gg m^{2}$, the hard $\left(\vec{p}_{g, T} \neq 0\right)$ contributions becomes sizeable and the quality of the NLL approximation becomes worse for both $\varphi$-dependent and $\varphi$-independent cross sections. Moreover, we have observed that softgluon approximation overestimates the exact results for $c_{T}^{(1,0)}(\eta, \xi)$ and $c_{T}^{(1,1)}(\eta, \xi)$ at $\xi \gg 1$ and, simultaneously, underestimates the corresponding ones for $c_{A}^{(1,1)}(\eta, \xi)$. For this reason, in the high- $Q^{2}$ region, the full NLO corrections to the $\cos 2 \varphi$ asymmetry may be smaller than the soft-gluon ones.

As to the energy dependence, one can see from Figs. 2 and 3 that soft radiation describes very well the exact NLO results on $\varphi$-independent photon-gluon fusion at partonic energies up to $\eta \approx 2$. Since the gluon distribution 
function supports just the threshold region, the soft-gluon contribution dominates the photon-hadron cross sections approximately up to $S / 4 m^{2} \sim 10$ (and, correspondingly, up to $\rho \sim 0.1$ for $\sigma_{l N}$ ). Using the exact results for the $\gamma^{*} g$ cross sections [28], we have verified that the contribution originating from the region $\eta>2$ makes only few percent from the NLO hadron-level predictions for $\sigma_{T}\left(S, Q^{2}\right)$ and $\sigma_{L}\left(S, Q^{2}\right)$ at $S / 4 m^{2} \lesssim 10$ (and $Q^{2} \lesssim m^{2}$ ). Results of Ref. [26] on soft-gluon corrections to $F_{2}^{\text {charm }}(x, \xi)$ confirm our conclusion.
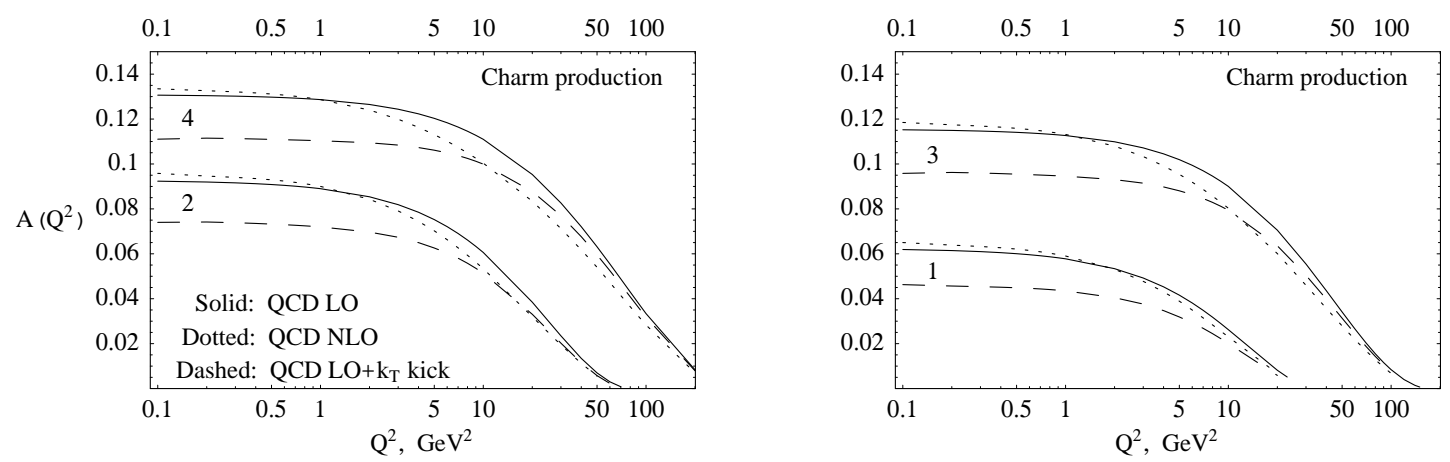

FIG. 4. Azimuthal asymmetry, $A\left(Q^{2}\right)$, in $c$-quark production for several values of initial energy: $E_{1}=24 \mathrm{GeV}, E_{2}=47$ $\mathrm{GeV}, E_{3}=95 \mathrm{GeV}$ and $E_{4}=190 \mathrm{GeV}$. Plotted are the results at LO (solid curve), at NLO to NLL accuracy (dotted curve) and at LO with $k_{T}$ smearing (dashed curve).
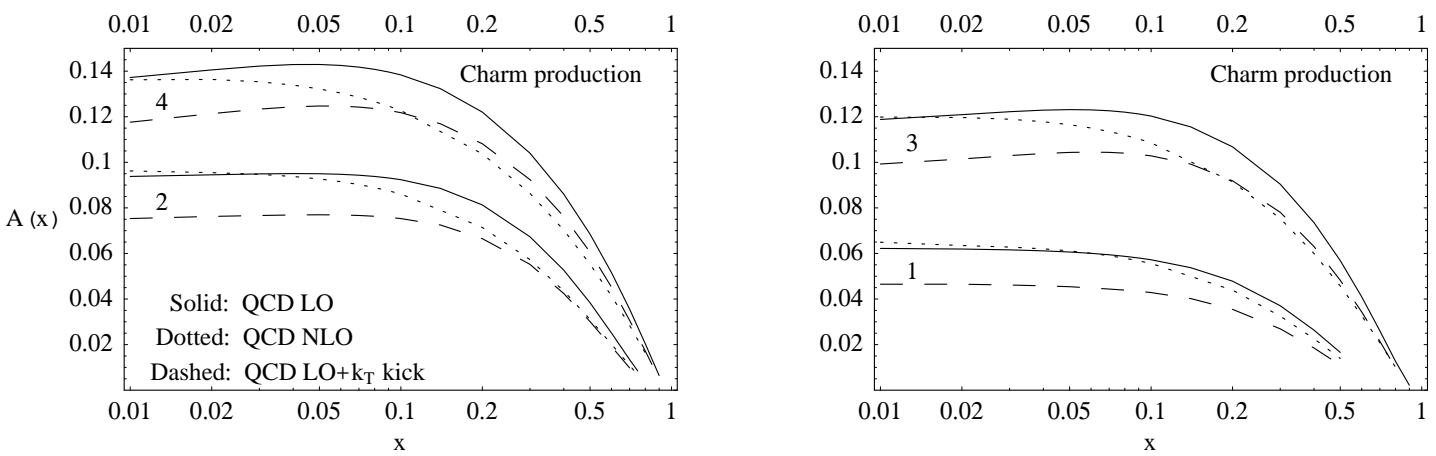

FIG. 5. Azimuthal asymmetry, $A(x)$, in $c$-quark production. The notation is the same as in Fig. 4.
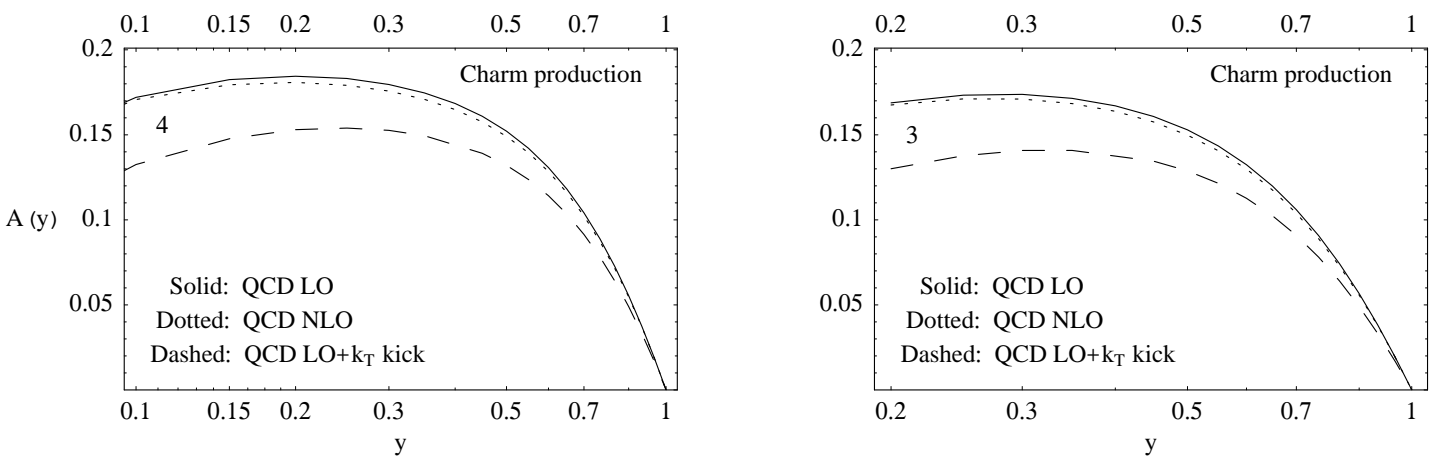

FIG. 6. Azimuthal asymmetry, $A(y)$, in $c$-quark production. The notation is the same as in Fig. 4. 

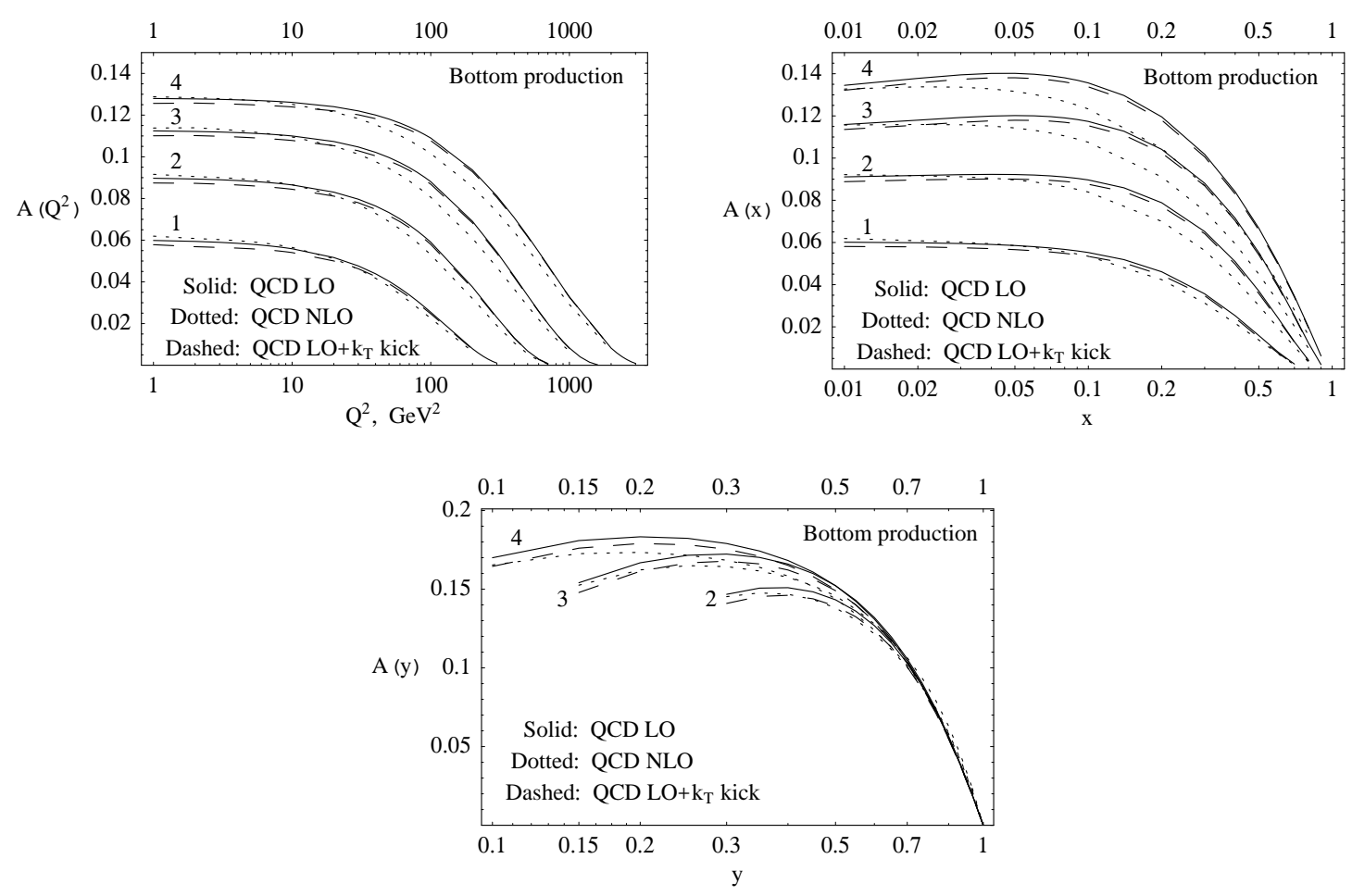

FIG. 7. Asymmetry parameters $A\left(Q^{2}\right)$ (left panel), $A(x)$ (right panel) and $A(y)$ (down panel) in $b$-quark production for several values of initial energy: $E_{1}=240 \mathrm{GeV}, E_{2}=480 \mathrm{GeV}, E_{3}=960 \mathrm{GeV}$ and $E_{4}=1920 \mathrm{GeV}$. Plotted are the results at LO (solid curve), at NLO to NLL accuracy (dotted curve) and at LO with $k_{T}$ smearing (dashed curve).
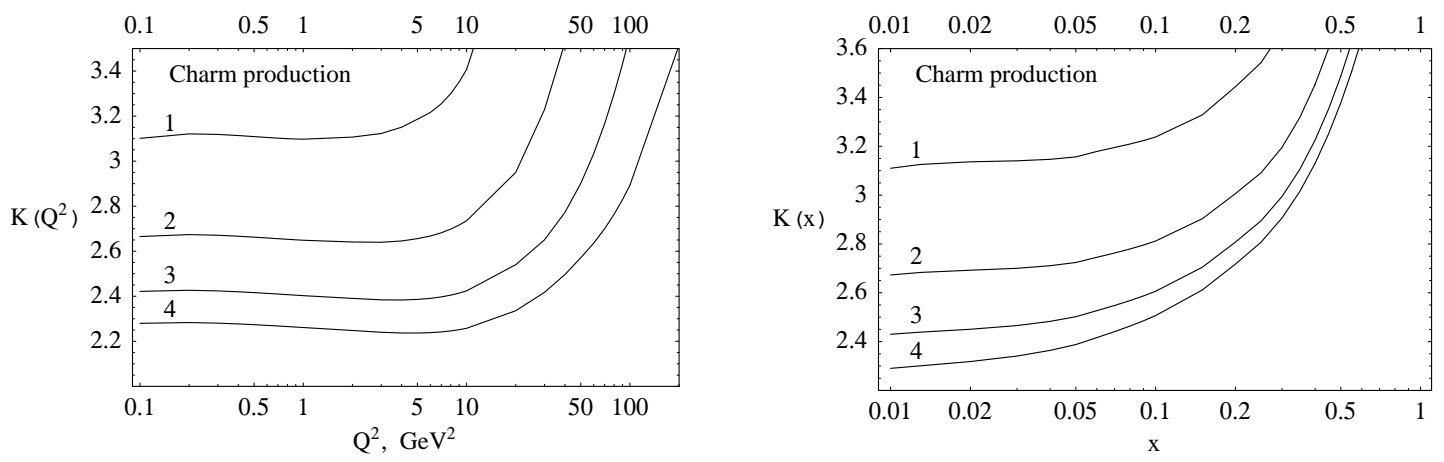

FIG. 8. $K$-factors in charm production at NLL level: $K\left(Q^{2}\right)=\frac{\mathrm{d} \sigma_{l N}^{\mathrm{NLO}}}{\mathrm{d} Q^{2}} / \frac{\mathrm{d} \sigma_{l N}^{\mathrm{LO}}}{\mathrm{d} Q^{2}}$ (left panel) and $K(x)=\frac{\mathrm{d} \sigma_{l N}^{\mathrm{NLO}}}{\mathrm{d} x} / \frac{\mathrm{d} \sigma_{l N}^{\mathrm{LO}}}{\mathrm{d} x}$ (right panel). The notation is the same as in Fig. 4.

Presently, exact NLO calculations of the $\varphi$-dependent cross section of heavy flavor production are not completed. However we can be sure that, at energies not so far from the production threshold, the soft radiation is the dominant perturbative mechanism in the case of $\sigma_{A}\left(S, Q^{2}\right)$ too. First, LO predictions for the cos $2 \varphi$-dependent cross section are large and the Sudakov logarithms have universal, $\varphi$-independent structure. For this reason, $\sigma_{A}\left(S, Q^{2}\right)$ has also a strong threshold enhancement. Second, our analysis of the exact scale-dependent $\operatorname{cross}$ section $c_{A}^{(1,1)}(\eta, \xi)$ given in Figs. 2 and 3 confirms with a good accuracy the dominance of the soft-gluon contribution. These facts argue that hard and virtual corrections to the $\cos 2 \varphi$-dependent cross section cannot affect significantly the soft-gluon predictions for the azimuthal asymmetry at low $Q^{2}$ in the energy region up to $S / 4 m^{2} \sim 10$. 

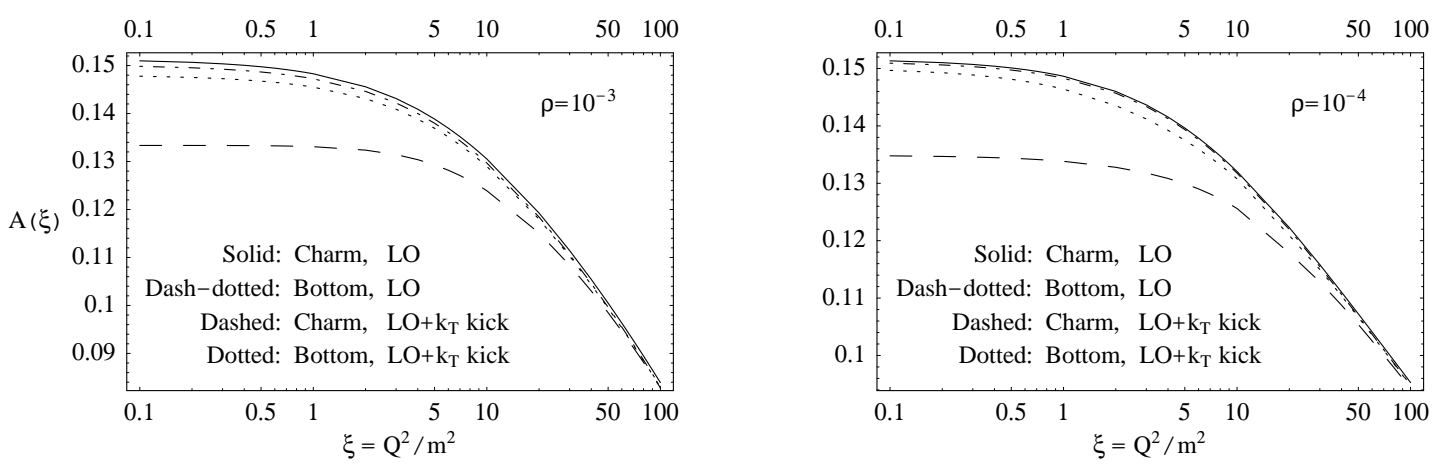

FIG. 9. QCD LO predictions for $A(\xi)$ in charm and bottom production at $\rho=10^{-3}$ (left panel) and $\rho=10^{-4}$ (right panel) with and without the inclusion of the $k_{T}$ smearing effect.

Let us briefly discuss the origin of perturbative stability of the $\cos 2 \varphi$ asymmetry. Note that the mere $\varphi$ independent structure of the Sudakov logarithms cannot explain our results since perturbative stability does not take place at the parton level. In fact, the ratios $\frac{c_{A}^{(1,0)}}{c_{T}^{(1,0)}}(\eta, \xi)$ and $\frac{c_{A}^{(0,0)}}{c_{T}^{(0,0)}}(\eta, \xi)$ differ essentially from each other even at $\eta, \xi \lesssim 1$. This is due to the fact that the physical soft-gluon corrections (2.8) are determined by a convolution of the Born cross sections with the Sudakov logarithms which, apart from factorized $\delta\left(s_{4}\right)$-terms, contain also nonfactorizable ones (see Eq. (2.12)). Kinematically, sizeable values of $\eta \sim 1$ allow $s_{4} / m^{2} \sim 1$ that leads to significant non-factorizable corrections. In other words, collinear bremsstrahlung carries away a large part of initial energy. Since the $\varphi$-dependent and $\varphi$-independent Born level partonic cross sections have different energy behavior, the soft radiation has different impact on these quantities.

Our analysis shows that two more factors are responsible for perturbative stability of the hadron level asymmetry. First, one can see from Figs. 2 and 3 that both $\varphi$-dependent and $\varphi$-independent Born level cross sections take their maximum values practically at the same values of $\eta$. Second, at fixed target energies, the gluon distribution function supports the contribution of the threshold region. In other words, sufficiently soft gluon distribution function makes the collinear gluon radiation effectively soft at the hadron level. In detail, the role of the gluon distribution function in perturbative stability of the azimuthal asymmetry in heavy quark photoproduction is discussed in Ref. [14].

Another remarkable property of the azimuthal asymmetry closely related to fast perturbative convergence is its parametric stability. ${ }^{3}$ Our analysis shows that the pQCD predictions for the $\cos 2 \varphi$ asymmetry are less sensitive to standard uncertainties in the QCD input parameters than the corresponding ones for the production cross sections. For instance, changes of $\mu_{F}$ in the range $m_{c}<\mu_{F}<2 \sqrt{m_{c}^{2}+Q^{2} / 4}$ affect the quantity $A\left(Q^{2}\right)$ in charm production by less than $7 \%$ at $\rho=0.025$ and $\xi \leq 4$. For the $\varphi$-integrated cross section, such changes of $\mu_{F}$ lead to $30 \%$ variations in the same kinematics. We have also verified that all the NLO CTEQ5 versions of gluon density as well as the LO parametrization [31] lead to asymmetry predictions which coincide with each other with an accuracy of better than $1.5 \%$.

Parametric stability of the azimuthal asymmetry leads to the scaling: with a good accuracy the quantity $A(\rho, x, \xi)$ in Eq. (1.6) is a function of three variables, so that

$$
A^{\text {Charm }}(\rho, x, \xi) \approx A^{\text {Bottom }}(\rho, x, \xi)
$$

at the same values of $\rho, x$ and $\xi$. To illustrate this property, in Fig. 9 we plot the asymmetry parameter $A\left(Q^{2}\right)$ defined by Eqs. (3.1) as a function of $\xi$ at $\rho=10^{-3}$ and $10^{-4}$. Since the soft-gluon approximation is inapplicable to heavy flavor production at high energies, we give only the LO predictions for $A(\xi)$. The LO results for the charm

\footnotetext{
${ }^{3}$ Of course, parametric stability of the fixed order results does not imply a fast convergence of the corresponding series. However, a fast convergent series must be parametrically stable. In particular, it must be $\mu_{R^{-}}$and $\mu_{F}$-independent.
} 
and bottom cases are plotted by solid and dash-dotted lines, respectively. One can see that both curves coincide with each other with an accuracy of better than $1 \%$.

It is also seen from Fig. 9 that, at the HERA energy $\left(\rho \sim 10^{-3}\right.$ and $10^{-4}$ for bottom and charm quark, respectively), the pQCD predictions for the AA in heavy quark leptoproduction are large and can be tested experimentally.

\section{B. Nonperturbative corrections}

Let us discuss how the pQCD predictions for azimuthal asymmetry are affected by nonperturbative contributions due to the intrinsic transverse motion of the gluon in the target. Because of the relatively low $c$-quark mass, these contributions are especially important in the description of the cross sections for charmed particle production [1].

To introduce $k_{T}$ degrees of freedom, $\vec{k}_{g} \simeq z \vec{p}+\vec{k}_{T}$, one extends the integral over the parton distribution function in Eq. (2.4) to $k_{T}$-space,

$$
\mathrm{d} z g\left(z, \mu_{F}\right) \rightarrow \mathrm{d} z \mathrm{~d}^{2} k_{T} f\left(\vec{k}_{T}\right) g\left(z, \mu_{F}\right) .
$$

The transverse momentum distribution, $f\left(\vec{k}_{T}\right)$, is usually taken to be a Gaussian:

$$
f\left(\vec{k}_{T}\right)=\frac{\mathrm{e}^{-k_{T}^{2} /\left\langle k_{T}^{2}\right\rangle}}{\pi\left\langle k_{T}^{2}\right\rangle} .
$$

In practice, an analytic treatment of $k_{T}$ effects is usually used. According to [32], the $k_{T}$-smeared differential cross section of the process (1.1) is a 2-dimensional convolution:

$$
\frac{\mathrm{d}^{4} \sigma_{l N}^{\text {kick }}}{\mathrm{d} x \mathrm{~d} Q^{2} \mathrm{~d} p_{Q T} \mathrm{~d} \varphi}\left(\vec{p}_{Q T}\right)=\int \mathrm{d}^{2} k_{T} \frac{\mathrm{e}^{-k_{T}^{2} /\left\langle k_{T}^{2}\right\rangle}}{\pi\left\langle k_{T}^{2}\right\rangle} \frac{\mathrm{d}^{4} \sigma_{l N}}{\mathrm{~d} x \mathrm{~d} Q^{2} \mathrm{~d} p_{Q T} \mathrm{~d} \varphi}\left(\vec{p}_{Q T}-\frac{1}{2} \vec{k}_{T}\right) .
$$

The factor $\frac{1}{2}$ in front of $\vec{k}_{T}$ in the r.h.s. of Eq. (3.5) reflects the fact that the heavy quark carries away about one half of the initial energy in the reaction (1.1).

Values of the $k_{T}$-kick corrections to LO predictions for the $\cos 2 \varphi$ asymmetry in the charm production are shown in Figs. $4-6$ and 9 by dashed curves. Calculating the $k_{T}$-kick effects we use $\left\langle k_{T}^{2}\right\rangle=0.5 \mathrm{GeV}^{2}$. At fixed target energies, $k_{T}$-smearing for $A\left(Q^{2}\right)$ and $A(x)$ is about $20-25 \%$ in the region of low $Q^{2}$ and $x$, respectively, and decreases at large $Q^{2}$ and $x$. In the HERA range, expected values of the $k_{T}$-corrections are systematically smaller (see Fig. 9).

Analogous calculations for the case of bottom production are presented in Figs. 7 and 9. It is seen that $k_{T}$-kick corrections to the $b$-quark AA are practically negligible in the whole region of $Q^{2}$ and $x$.

\section{CONCLUSION}

In this paper we have investigated the impact of soft-gluon radiation on the $\cos 2 \varphi$ asymmetry in heavy flavor leptoproduction. The NLL approximation provides a good description of the available exact NLO results for $Q^{2} \lesssim$ $m^{2}$ at energies of the fixed target experiments. Our calculations show that the azimuthal asymmetry is practically insensitive to soft-gluon corrections in this kinematics. We conclude that, unlike the $\varphi$-integrated cross sections, the $\cos 2 \varphi$ asymmetry in heavy quark leptoproduction is an observable quantitatively well defined in pQCD: it is stable both parametrically and perturbatively, and insensitive (in the case of bottom production) to nonperturbative contributions. This asymmetry is of leading twist and predicted to be about $15 \%$ at energies sufficiently above the production threshold for both charm and bottom quark. Measurements of the $\cos 2 \varphi$ asymmetry in bottom production at HERA would provide an ideal test of pQCD.

Data on the charm azimuthal distributions from the COMPASS and HERMES experiments would make it possible to clarify the role of subleading twist contributions. Our analysis shows that, in the low- $x$ region, the AA is sensitive to the gluon transverse motion in the target. At high $x$, the intrinsic charm contribution [33] to the structure functions may be significant $[34,35]$. In detail, the possibility of measuring the intrinsic charm content of the proton using the $\cos 2 \varphi$ asymmetry will be considered in a forthcoming publication. 


\section{ACKNOWLEDGMENTS}

The author would like to thank S.J. Brodsky, A.B. Kaidalov, A.Kotzinian and A.G. Oganesian for useful discussions. I am grateful to High Energy Section of ICTP for hospitality while this work has been completed.

[1] S. Frixione, M.L. Mangano, P. Nason and G. Ridolfi, published in: Heavy Flavours II, A.J. Buras and M. Lindner (Eds.), Advanced Series on Directions in High Energy Physics, Vol. 15, World Scientific, Singapore, $1998 ;$ hep-ph/9702287.

[2] M.L. Mangano, P. Nason and G. Ridolfi, Nucl. Phys. B373 (1992), 295.

[3] S. Frixione, M.L. Mangano, P. Nason and G. Ridolfi, Nucl. Phys. B412 (1994), 225.

[4] N. Kidonakis, Int. J. Mod. Phys. A15 (2000), 1245.

[5] H. Contopanagos, E. Laenen and G. Sterman, Nucl. Phys. B484 (1997), 303.

[6] E. Laenen, G. Oderda and G. Sterman, Phys. Lett. B438 (1998), 173.

[7] N. Kidonakis, G. Oderda and G. Sterman, Nucl. Phys. B531 (1998), 365.

[8] E. Laenen, J. Smith and W.L. van Neerven, Nucl. Phys. B369 (1992), 543.

[9] E.L. Berger and H. Contopanagos, Phys. Rev. D 54 (1996), 3085.

[10] S. Catani, M.L. Mangano, P. Nason and L. Trentadue, Nucl. Phys. B478 (1996), 273.

[11] N. Kidonakis, Phys. Rev. D64 (2001), 014009.

[12] H. Lai and H. Li, Phys. Lett. B471 (1999), 220.

[13] N.Ya. Ivanov, A. Capella and A.B. Kaidalov, Nucl. Phys. B586 (2000), 382.

[14] N.Ya. Ivanov, Nucl. Phys. B615 (2001), 266.

[15] N.Ya. Ivanov, P.E. Bosted, K. Griffioen and S.E. Rock, Nucl. Phys. B650 (2003), 271.

[16] SLAC E161, (2000), http://www.slac.stanford.edu/exp/e160.

[17] N. Dombey, Rev. Mod. Phys. 41 (1969), 236.

[18] J.P. Leveille and T. Weiler, Phys. Rev. D24 (1981), 1789.

[19] A.D. Watson, Zeit. Phys. C12 (1982), 123.

[20] J.P. Leveille and T. Weiler, Nucl. Phys. B147 (1979), 147.

[21] E. Laenen, S. Riemersma, J. Smith and W.L. van Neerven, Nucl. Phys. B392 (1993), 162.

[22] R.K. Ellis and P. Nason, Nucl. Phys. B312 (1989), 551.

[23] J. Smith and W.L. van Neerven, Nucl. Phys. B374 (1992), 36.

[24] P. Nason, S. Dawson and R.K. Ellis, Nucl. Phys. B303 (1988), 607; Nucl. Phys. B327 (1989), 49; Erratum: ibid B335 (1990), 260.

[25] W. Beenakker, H. Kuijf, W.L. van Neerven and J. Smith, Phys. Rev. D 40 (1989), 54.

[26] E. Laenen and S.-O. Moch, Phys. Rev. D 59 (1999), 034027.

[27] T.O. Eynck and S.-O. Moch, Phys. Lett. B495 (2000), 87.

[28] S. Riemersma, J. Smith and W.L. van Neerven, Phys. Lett. B347 (1995), 43; B.W. Harris and J. Smith, Nucl. Phys. B452 (1995), 109.

[29] W. Furmanski and R. Petronzio, Phys. Lett. B97 (1980), 437.

[30] H.L. Lai et al., Eur. Phys. J. C12 (2000), 375.

[31] A. Capella, A.B. Kaidalov, C. Merino and J. Tran-Thanh Van, Phys. Lett. B337(1994), 358.

[32] L. Apanasevich, C. Balazs, C. Bromberg et al., Phys. Rev. D59 (1999), 074007.

[33] S.J. Brodsky, P. Hoyer, C. Peterson and N. Sakai, Phys. Lett. B93 (1980), 451;

S.J. Brodsky, C. Peterson and N. Sakai, Phys. Rev. D 23 (1981), 2745.

[34] E. Hoffmann and R. Moore, Zeit. Phys. C20 (1983), 71.

[35] B.W. Harris, J. Smith and R. Vogt, Nucl. Phys. B461 (1996), 181. 